\title{
LA CAPACIDAD PREDICTIVA EN EL RENDIMIENTO ESCOLAR DEL CAPITAL ECONÓMICO Y CULTURAL DE LAS FAMILIAS DEL ESTUDIANTADO EN ESPAÑA Y MARRUECOS
}

The economic and cultural capitals of families as predictors of student performance in Spain and Morocco

Miguel Ángel LOZANO PÉREZ

Universidad de Granada

lozanop@correo.ugr.es

http://orcid.org/0000-0002-7262-3405

Antonio TRINIDAD REQUENA

Universidad de Granada

atrinida@ugr.es

https://orcid.org/0000-0002-3075-0983

Recibido: 18/10/2019. Revisado y aceptado para publicación: 09/12/2020

Para citar este artículo: Miguel Ángel LOZANO PÉREZ y Antonio TRINIDAD REQUENA (2020), "La capacidad predictiva en el rendimiento escolar del capital económico y cultural de las familias del estudiantado en España y Marruecos" en Revista de Estudios Internacionales Mediterráneos, 29,pp. 130-151.

Para acceder a este artículo: https://doi.org/10.15366/reim2020.29.010

\section{Resumen}

Las principales evaluaciones internacionales en el campo de la educación ponen de manifiesto que el origen familiar influye en el rendimiento escolar y que el nivel de relación de estas variables varía según el país. Por lo que surge la siguiente cuestión: ¿Cómo opera la desigualdad de oportunidades educativas según el origen familiar?. Para responder a esta pregunta hemos analizado dos sociedades que componen una de las fronteras más desiguales del mundo: España y Marruecos. Nuestro planteamiento es que el capital económico y el cultural poseen un efecto acumulativo en la desigualdad de oportunidades educativas. Sin embargo, el mayor desarrollo socioeconómico conlleva que el capital cultural sea mejor predictor en España que en Marruecos, mientras el origen económico de la familia tiene mayor peso en el país magrebí. Para testar esta hipótesis hemos analizado los datos de PIRLS 2016. Los resultados han sido acordes con la hipótesis, pero con mayor margen en el caso marroquí que en el español.

Palabras clave: capital cultural/capital económico/rendimiento escolar/Marruecos/España 


\begin{abstract}
Leading international assessments in the field of education show that family origins influence student performance, and that the degree of relation between these variables differs from one country to another. This raises the following question: how do family origins contribute to inequality of opportunities in education? To answer this question, we analyse two societies located on both sides of one of the world's most unequal borders: Spain and Morocco. We posit that economic and cultural capitals have a cumulative effect on inequality of opportunities in education. However, the higher level of socio-economic development makes cultural capital a better predictor in Spain than in Morocco, while the economic background of families carries greater weight in Morocco. To test this hypothesis we analyse data from PIRLS 2016. Our findings bear out the hypothesis, but with a higher support for Morocco than for Spain.
\end{abstract}

Keywords: cultural capital/economic capital/ student performance/Morocco/Spain

\title{
Introducción
}

\section{El capital económica y el cultural de la familia estudiantado como predictores del rendimiento escolar}

A partir de la propuesta de Bourdieu, tanto en solitario $(1979 ; 2011)$ como junto con Passeron (1964), testaremos con datos de PIRLS 2016 la siguiente hipótesis: (H1) las características económicas de los progenitores son mejores predictores del rendimiento educativo en Marruecos que en España, ya que en este segundo país poseen mayor peso el capital cultural de la familia.

Los principales informes internacionales encargados de evaluar el rendimiento educativo ponen de manifiesto que el origen familiar influye en los resultados del alumnado, ya sea PIRLS (Progress in International Reading Literacy Study) (Mullis et al., 2017), TIMSS (Trends in International Mathematics and Science Study) (Martin et al., 2016; Mullis et al., 2016) o PISA (Programme for International Student Assessment) (OECD, 2016a; 2016b). Sin embargo, si vamos más allá, nos preguntamos cómo se produce este fenómeno. $Y$ ahí aparecen ciertas divergencias, lo que ha dado lugar a diferentes teorías, técnicas de análisis y, también, resultados (Fernández, 2015). Lo cual indica que en diferentes contextos dicho fenómeno se articula de diferente forma. Por lo que, cabe esperar, en distintas sociedades el origen del estudiantado influya en su rendimiento con diferente fuerza y forma. Por tanto, si dos países diferentes poseen poblaciones diferentes en lo económico, social y cultural deberíamos esperar que la influencia del origen familiar en el rendimiento escolar también lo fuese. Los anteriores informes ponen de manifiesto que existe tal diferencia, pues a mayor desarrollo socioeconómico mayor rendimiento y a menores desigualdades socioeconómicas menores diferencias de rendimiento escolar según el entorno del estudiantado. Sin embargo, surgen una cuestión: ¿cómo opera la desigualdad de oportunidades educativas en la escuela para que así suceda?

Existen dos perspectivas enfrentadas, en los planos teórico y empírico, sobre cómo tiene lugar este fenómeno (De Graff et al., 2000; Álvarez-Sotomayor y Martínez-Cousinou, 2016): la de Raymond Boudon (1974), donde la principal causa es el coste de oportunidad, y la de Pierre Bourdieu (2011), centrada en la reproducción cultural. Sin embargo, nuestra propuesta es una relectura de Bourdieu, en la que el capital cultural emerge cuando las oportunidades formales, o económicas, son suavizadas por el desarrollo económico o del Estado de Bienestar.

Así nuestra hipótesis viene configurada con la siguiente lógica. En una economía poco desarrollada 
y con un estado de bienestar débil el factor económico de la familia es crucial, ya que es una barrera manifiesta para poder acceder a una serie de servicios y recursos en el campo educativo. Por contraposición, en una economía más desarrollada y con un estado de bienestar más fuerte la situación económica de la familia pierde poder explicativo, mientras los factores culturales de esta ganan preponderancia al explicar el rendimiento escolar. No estamos afirmando que el bagaje cultural de una familia solo se manifieste en igualdad económica, estamos manteniendo que su capacidad predictiva es más manifiesta.

Esta cuestión ya fue planteada por Pierre Bourdieu, tanto con Passeron (1964), como en solitario (1979; 2011). Sin embargo, no lo hizo para explicar estas diferencias de rendimiento en distintos territorios, a excepción de la dualidad urbano/rural. La utilizó para explicar el porqué en la Francia de la década de 1960 en adelante persistían diferencias según el origen del estudiantado, pese al desarrollo de planes de becas y ayudas al estudio.

Pero antes, para abordar mejor la lógica que subyace a este planteamiento es necesario distinguir tres conceptos: capital, campo y habitus. Bourdieu entiende por capital cierta ventaja que poseen los individuos a la hora de competir en diferentes escenarios. Así, a pesar de que no acota estrictamente el número de capitales, Bourdieu $(1979 ; 2011)$ distingue tres principales como factores explicativos: económico, social y cultural.

Siguiendo con la lógica expuesta en estas obras, los capitales poseen diferente importancia según el espacio social donde se desarrollan, a estos espacios los denomina campos. Por consiguiente, en el mundo de los negocios el capital económico es fundamental, mientras en la escuela el capital cultural es aquel que mayor preponderancia posee. Así una persona que pertenece a una familia adinerada tendrá más oportunidades de abrir su propio negocio, mientras otra persona con ascendentes con niveles culturales más altos tendrá mayor probabilidad de desenvolverse en el sistema educativo.

Por último, el habitus son una serie de prácticas sociales que permiten mutar entre los diferentes capitales (Martínez, 2017). Así una persona con mayor capital educativo puede acceder a un mejor empleo y, por tanto, a mayor capital económico. Mientras otra con un mayor capital económico puede acceder a prácticas sociales o formación que generen un mayor capital cultural.

En lo que a nuestro objeto de estudio se refiere, Bourdieu y Passeron (1964) entendían que el capital económico y el cultural eran acumulativos, sin embargo, por muchos esfuerzos que hiciera el estado por igualar los mecanismos del primero de estos en el campo educativo iban a persistir diferentes habitus heredados del capital cultural, tal y como expusieron:

Es así como los mecanismos que aseguran la eliminación de los niños de clases bajas y media actuarían casi con la misma eficacia (pero más discretamente) en el caso en el que una política sistemática de becas o subsidios de estudio volviera formalmente iguales ante la educación a los sujetos de todas las clases sociales. (Bourdieu y Passeron, p.44).

Un punto que necesita ser tratado es la especificidad del capital cultural heredado. En el campo educativo, Bourdieu lo acota a las prácticas sociales en las que el individuo es sociabilizado en el seno de una familia, y que conllevan una ventaja competitiva en las instituciones escolares.

Por último, hay tres cuestiones que aclarar. La primera es que hemos ignorado al capital social porque no influye en nuestra hipótesis, debido a que este capital se hereda en menor medida entre distintas generaciones, pues las familias pueden generarlo con mayor facilidad que los otros dos a partir de diferentes estrategias (Álvarez-Sotomayor, 2012). La segunda cuestión es que en nuestra hipótesis no se tiene en consideración la importancia del momento en la trayectoria vital del estudiantado, pese a la existencia de evidencia que demuestra que las becas o subsidios no son efectivos porque la intervención es más eficiente cuando es temprana (Carneiro y Heckman, 2003). La última es que no pretendemos medir la influencia del estado y de sus políticas para 
suavizar las diferencias educativas de origen.

\section{La frontera Marruecos-España: una frontera desigual en el campo educativo}

La frontera marroquí-española se configura como una de las más desiguales que existen en el planeta. Así ambos países ya han sido objeto de estudio por configurar territorios fronterizos profundamente asimétricos entre ellos (Trinidad et al., 2019). Tanto es así, que el PIB per cápita a precios actuales es nueves veces mayor en España, siendo 28.208,3 US\$ por 3.022,9 en el país magrebí (Banco Mundial ${ }^{1}$ ), situados en las posiciones 37a y 136a. Lo cual se refleja, también, en el IDH. Ocupando el país ibérico el puesto 26 (IDH: 0,891) y Marruecos el $123(0,667)$, según la ONU². Respecto a la desigualdad interna de cada uno de estos países los datos también son favorables para España. Ya que los valores del índice de Gini eran 36,2 (para 2015) y 39,5 (2013), según los últimos datos del Banco Mundial ${ }^{3}$. Como indica Moré (2003), esta frontera es una de las 10 más desiguales del mundo.

Respecto al sistema educativo, la educación primaria comienza en ambos países a los seis años con una duración de seis cursos. Sin embargo, las diferencias se centran en las etapas anteriores y posteriores. La etapa anterior de tres cursos es gratuita en España, en contraposición en Marruecos es privada, teniendo una duración de cuatro cursos. La etapa posterior a primaria y última con carácter obligatorio en Marruecos es de tres cursos, en España se prolonga un curso más. Lo cual conlleva que en el país europeo la educación secundaria post-obligatoria tenga un curso menos, siendo de dos. La formación profesional de mayor cualificación es de dos cursos en ambos países. Donde sí encontramos diferencias es en el ciclo de un nivel menor, teniendo tres cursos en Marruecos, lo cual es un curso más que en España. Por último, la educación universitaria en España son cuatros cursos de grado más uno de máster y en Marruecos son tres de licenciatura y dos de máster. En este aspecto, cabe señalar que aunque las ayudas y becas son menores en Marruecos, las universidades públicas son totalmente gratuitas ${ }^{4}$.

Respecto a las diferencias educativas, el reino alauí posee una tasa de alfabetización ${ }^{5}$ del $69,43 \%$, mientras en España es del 97,89\%. Siendo los últimos datos comparables de 2012, esto.

Atendiendo a los resultados del estudio realizado por la IEA (International Association for the Evaluation of Educational Achievement) sobre comprensión lectora, nos encontramos dos conclusiones principales. La primera es que el rendimiento es mayor en el país ibérico que en el

\footnotetext{
${ }^{1}$ The World Bank, Popular indicators, disponible en https://databank.worldbank.org/indicator/NY.GDP.MKTP.CD/1ff4a498/Popular-Indicators [consulta: 30 de mayo de 2019]

${ }^{2}$ United Nations Development Programe, ONU, Human Development Data (1990-2017), disponible en http://hdr.undp.org/en/data\# [consulta: 30 de mayo de 2019]

${ }^{3}$ Tanto los datos de la ONU como los del Banco Mundial (The World Bank en inglés) son de 2017, salvo cuando está indicado entre paréntesis. Además, todos estos datos se encuentran en la dirección de los comentarios a pie de página 1 y 2.

${ }^{4}$ Para más información recúrrase a las fuentes: BOLETín OFICIAL DEL ESTADO (2013). Ley Orgánica, de 9 de diciembre, para la Mejora de la Calidad Educativa, disponible en https://www.boe.es/diario boe/txt.php?id=BOE-A-2013-12886 [consulta : 12 de abril de 2020] ; COMMISSION SPÉCIAL EDUCATION FORMATION (1999): Charte Nationale d'Éducation et de Formation, disponible en

http://www.mcinet.gov.ma/sites/default/files/documentation\%20iscae\%20rabat\%202018.pdf [consulta: 12 de Abril de 2020].

${ }^{5}$ UNESCO, Taux d'alphabétisme, disponible en http://data.uis.unesco.org/?lang=fr\&SubSessionld=4b11b2d8-fa504db1-a09e-ca1ec612a1f8\&themetreeid=-200 [ consulta: 30 de Mayo de 2019]
} 
magrebí, con unas puntuaciones medias de 528 y 358 respectivamente. La segunda constata que el entorno social, cultural y económico del estudiantado influye en el rendimiento del alumnado de los diferentes países. Y, por consiguiente, en España existe un mayor capital económico, cultural y social que se traduce en un mayor rendimiento en las pruebas. En este aspecto el Informe PIRLS 2016 (Mullins et al., 2017) presenta diferentes variables y análisis, nunca sobrepasando el análisis bivariante. Además, el INEE (Instituto Español de Evaluación Educativa) genera un informe para el caso español (2017), donde se amplían estos resultados, aunque en los países con los que compara a España ignora a Marruecos por no pertenecer a la UE ni a la OCDE.

Esta información es redundante con otros estudios. Así, Fasfous et al. (2013) defienden que las diferencias culturales ${ }^{6}$ son tan grandes entre estos dos países que no se puede comparar test de inteligencia de ambas poblaciones. Soriano-Miras et al. (2015) van más allá. Ya que no solo defienden que las desigualdades de oportunidades educativas son mayores en Marruecos que en España, además ponen de manifiesto que en los países desarrollados la desigualdad educativa es inferior a la desigualdad general, mientras en las economías emergentes la desigualdad educativa es mayor a la mayoría de las desigualdades.

En esta línea, Bijou y Bennouna (2018) advierten como en Marruecos el aumento de la inversión en educación por parte del Estado no ha conseguido disminuir significativamente la fuerte influencia en el rendimiento escolar. Además, Oxfam (2019) señala que, pese a que en Marruecos la inversión estatal en educación es mayor que a la del resto de países del Magreb, las desigualdades educativas no son menores que la de dichos estados. Khander et al. (1994) advirtieron que la inversión en educación estaba siendo ineficiente debido a la ausencia de infraestructuras básicas como trasportes o electricidad, siendo más aguda esta problemática en las zonas rurales del país magrebí. En este aspecto, cabe señalar la existencia de estudios en los que distintos países presentan niveles de desigualdad interna diferentes (Fernández, 2015; Hanushek et al., 2010), aunque estos no incluyen países tan desiguales como los de nuestro objeto de estudio. Dichas diferencias se deben a la multitud de variables que poseen influencia en las desigualdades educativas en general y según el origen familiar de forma más específica. En consecuencia, para testar cómo operan el capital económico y el cultural de las familias del estudiantado en países con diferente nivel de desarrollo consideramos que España y Marruecos presentan diferencias suficientemente agudas.

\section{Metodología}

\subsection{La fuente: PIRLS 2016}

Hemos utilizado los datos de PIRLS $2016^{7}$. Este estudio es realizado por la IEA, y tiene como objeto de estudio conocer la comprensión lectora de niños y niñas de 40 grado ${ }^{8}$, según la clasificación internacional de la OECD (1999). Es decir, alumnado que en idoneidad de curso cumple nueve años en el año en el que se inicia el curso. Siendo la media de edad de 9,9 para la muestra española y 10,2 para las marroquíes. En este punto, es necesario hacer la advertencia de que la muestra es sobre quienes están escolarizados. $Y$ estos países presentan cifras diferentes de absentismo escolar, siendo de $5,42 \%$ en Marruecos y de $1,47 \%$ en España ${ }^{9}$. Estos datos no solo limitan la capacidad predictiva del estudio, además vuelven a poner de manifiesto las diferencias de oportunidades en ambos países. La elección de la base de datos de PIRLS se debe a que pocos estudios internacionales incluyen a Marruecos, junto a que la educación obligatoria marroquí

\footnotetext{
${ }^{6}$ En este caso los autores utilizan el concepto cultural con mucha más amplitud que nosotros, incluyendo factores que Bourdieu entendería propios del capital social.

${ }^{7}$ Los datos han sido obtenidos de https://www.iea.nl/data [consulta 12 de mayo de 2019]

${ }^{8}$ Denominado en el sistema educativo español "40 Primaria" y en el marroquí "4ㅇ de Escuela primaria".

${ }^{9}$ Datos del 2016. UNESCO, disponible en http://data.uis.unesco.org/Index.aspx?DataSetCode=edulit ds [ consulta: 30 de mayo de 2019]
} 
termina un curso antes que en España. Además, el abandono escolar prematuro demanda que no se haga sobre cursos muy avanzados.

Para nuestro estudio hemos utilizado tres muestras. Una española, que denominaremos España PIRLS y dos marroquíes: Marruecos PIRLS y Marruecos LP. El motivo para utilizar dos muestras marroquíes es el siguiente. PIRLS posee tres pruebas distintas con muestras, también, diferenciadas por cada país: PIRLS, e-PIRLS y PIRLS Literacy. La primera es la común a todos los países miembros. La siguiente la prueba es realizada con un ordenador, ninguno de los dos países objeto de estudio posee una muestra que haya realizado esta evaluación. Y la tercera se ha efectuado en países que poseen tasas de alfabetización muy bajas. Por lo que en Marruecos se ha seleccionado una muestra a la que se le ha realizado esta prueba, además de la común al resto, tal y como bien explica Foy (2018). En este punto, es importante destacar que PIRLS Literacy contiene cuestiones más sencillas que las otras dos pruebas, pues está diseñado para evaluar países con bajas tasas de alfabetización. También, aclarar, que en el caso español existen otras dos muestras, específicas para Andalucía y la Comunidad de Madrid, las cuales no las hemos utilizado al ser la muestra conjunta suficientemente representativa, véase tabla 1.

Aun cabiendo la posibilidad de analizar conjuntamente las dos bases de datos marroquíes formando una sola, hemos decidido distinguirlas por varias razones. La primera es que ambas muestras son suficientemente representativas (Tabla 1). Además, como hemos advertido la evaluación de Marruecos $L P$ es más sencilla que la de las otras dos muestras. Así Marruecos $L P$ tiene una puntuación media de 366 (DT: 102), España PIRLS de 528 (61) y Marruecos PIRLS de 349 (101). Por lo que podemos afirmar que el hecho de que el informe de la IEA (Mullis et al, 2017) presente conjuntamente los datos de los dos bases de datos existentes para Marruecos, pese a que su guía metodológica (Foy, 2018) advierte de que Marruecos LP posee una prueba más sencilla, es un error. Y que, por tanto, la media marroquí que arroja esta evaluación, concretamente de 358 , está sesgada.

Existe otra cuestión que diferencia las bases de datos de ambos países, hay una serie de preguntas de los cuestionarios que varían según el país, lo cual nos dificultará la selección de las variables, como veremos a continuación.

Las cuatro primeras filas de la Tabla 1 detallan a la muestra sin la eliminación de ningún individuo. Observando el tamaño de las tres muestras y a la población que representa, ya que en la propia base de datos existe una variable de ponderación para poder estimar el total de estudiantes de cuarto grado de cada país: TOTWGT. El peso de la muestra seleccionada se ha calculado a partir de otra variable (SENWGT) para que cada individuo de la muestra tenga su propio peso en el conjunto de esta. Por este motivo los individuos de Muestra seleccionada (fila 5) y Peso de la muestra seleccionada (fila 6) no son coincidentes, pues como detallamos a continuación se ha eliminado, aproximadamente, un tercio de la muestra. 
Tabla 1. Distribución de las tres submuestras.

\begin{tabular}{|l|c|c|c|}
\hline & $\begin{array}{l}\text { Marruecos } \\
\text { LP }\end{array}$ & España PIRLS & Marruecos PIRLS \\
\hline Muestra & 5453 & 14595 & 5489 \\
\hline Población & 664737 & 457645 & 664737 \\
\hline Nivel de confianza & $95 \%$ & $95 \%$ & $95 \%$ \\
\hline Error muestral & $\pm 1,3 \%$ & $\pm 0,8 \%$ & $\pm 1,3 \%$ \\
\hline Muestra seleccionada & 3552 & 9724 & 3520 \\
\hline Peso de la muestra seleccionada & 3617 & 9733 & 3658 \\
\hline Población seleccionada & 443355 & 305190 & 443028 \\
\hline Nivel de confianza & $95 \%$ & $95 \%$ & $95 \%$ \\
\hline Error muestral & $\pm 1,6 \%$ & $\pm 1 \%$ & $\pm 1,6 \%$ \\
\hline
\end{tabular}

Fuente: Elaboración propia a partir de datos de PIRLS 2016

\subsection{Las variables}

Para evaluar el rendimiento, lo que configura nuestra variable explicada, utilizaremos la propia evaluación de PIRLS 2016. Sin embargo, tenemos en consideración las diferencias entre las tres bases de datos. Primero, la cuestión de que las dos evaluaciones marroquíes son diferentes y segundo que el alumnado español tiene un rendimiento más alto que el marroquí. Por lo que la manera más eficiente para que esto no incida en los resultados es distinguir el rendimiento dentro de cada muestra. Así, hemos generado una variable denominada Rendimiento Prueba $(R P)$. Donde distinguimos al tercio del alumnado con mejor nota, Alto Rendimiento $(A R)$, de aquel tercio que peor calificación posee, Bajo Rendimiento $(B R)^{10}$. Este método posee la ventaja de que elimina los casos intermedios. Lo cual, para el caso español, ya se ha realizado en otros estudios (Pérez et al., 2013; García-Fernández et al., 2013; Lozano y Trinidad, 2019). Por lo que igualando la variable dependiente se eliminan las diferencias educativas entre ambos países, incluyendo el retorno de la inversión estatal en educación.

Para medir las variables explicativas, tanto el capital económico como el cultural, hemos seleccionado dos variables por cada uno de estos capitales. A estas cuatro hemos añadido otras dos que consideramos más intermedias, es decir que indican la acumulación de ambos capitales. Quedando repartidas en tres variables politómicas, que puedan interpretarse de forma ordinal, y otras tres numéricas, que se puedan recodificar en intervalos cuando fuese necesario. Además, se han respetados dos maneras de proceder en la recodificación. La primera es que todas las reagrupaciones siempre se han efectuado para tres categorías válidas, para un mejor análisis comparativo. Mientras, cuando el análisis requeriría el reagrupamiento de las variables numéricas, lo hemos efectuado dentro de cada base de datos ${ }^{11}$. Para poder comparar las diferencias de rendimiento según cada uno de los capitales en cada muestra, atendiendo únicamente a la distribución interna. $Y$ de este modo, inferir en qué poblaciones son mayores las diferencias según cada uno de los capitales. Además, hemos seleccionado cuatro variables de contexto: dos politómicas y otras tantas numéricas. Sin embargo, para estas cuatro variables no hemos respetado los anteriores requisitos, pues nos limitaba en exceso la selección y no las vamos a utilizar en el análisis final.

\footnotetext{
${ }^{10}$ Aunque las pruebas son muy amplias, hay alumnado que obtiene la misma calificación. Cuando varios individuos poseen la misma nota, estando en el límite de alguno de estos grupos, hemos decidido incluirlos a todos ellos.

${ }^{11}$ Esta cuestión es muy importante, porque en la base de datos de PIRLS, las variables politómicas que son reagrupaciones a partir de rangos de los índices siempre se construyen a partir del conjunto de datos de todos los países, aunque trabajes con una muestra de un solo país.
} 
Para el capital cultural de la familia hemos seleccionado como variable politómica el Número de libros en el hogar (Libr_Hoga) por dos razones. La primera es de carácter teórico, y es que esta variable es utilizada, expresamente, por Bourdieu $(1964 ; 1979)$ para medir el capital cultural, mientras el padre del capital social no la utiliza en los análisis de este (Coleman, 1968). La segunda, de carácter metodológico, es que en PIRLS esta cuestión se le realiza al padre o la madre con cinco categorías de respuesta ya determinadas, por lo que no se podía utilizar como numérica. Por lo que la hemos recodificado en de 0 a 10, de 11 a 100 y más de 100. Como variable continua hemos seleccionado uno de los seis índices que genera PIRLS según la familia de origen, a partir de varias cuestiones: Gusto por la lectura de ambos padres (Gust_Lect_scl).

La variable Politómica que hemos utilizado para medir el capital económico es la Ocupación más alta de ambos padres (Ocup_PyM). Si bien, esta está relacionada con el capital cultural y social, es la variable que mejor testa el capital económico de las que podemos utilizar, pues como señala García (2013) la ocupación mantiene relación con el nivel de ingresos, variable no incluida en PIRLS 2016. Ya que, comparativamente con PISA, PIRLS posee muchas menos cuestiones económicas, y aquellas que posee se diferencian en exceso de España a Marruecos. Por lo que PIRLS no pregunta ni por el número de coches ni de habitaciones en el hogar, y preguntando por el teléfono móvil en Marruecos lo hace sobre si se posee alguno en el hogar, mientras en España se atañe únicamente a si lo tiene el estudiante. Las categorías laborales las hemos construido a partir de la clasificación ISCO-08 (ILO, 2012). Quedando como categorías de respuesta: Trabajadores no cualificados (nivel 9), Trabajadores cualificados (4 a 8) y Empresarios y profesionales (1 a 3). Además, en la primera de las categorías se han incluido a quienes no trabajan. La variable continua seleccionada ha sido el índice, creado por la IEA, Dispositivos digitales en el hogar (Dip_Digt_scl). El cual está creado a partir de diferentes cuestiones, sobre si se posee móvil, ordenador personal, TV etc. Esta variable no debe confundirse con otras similares que poseen otros estudios internacionales como PISA, pero que lo que realmente miden son las nuevas tecnologías (Lozano y Trinidad, 2019).

Respecto a aquellas dos variables que hemos denominado como acumulativas hemos elegido las siguientes. Como variable ordinal el Nivel de estudios más alto de ambos padres (Estud_PyM). Es cierto que Bourdieu recurre a ella para medir el capital cultural en todas sus obras aquí citadas. Sin embargo, en La distinction: critique sociale du jugement (pp. 17-115, 1979) aclara que el nivel de estudios reconocidos posee un carácter credencialista que posibilita la transformación del capital cultural en capital económico, por lo que procede a denominar al nivel de estudios como capital escolar. Ya que el nivel de estudios más alto de ambos padres habilita para determinadas ocupaciones, indicando en menor grado el capital cultural que el número de libros en el hogar, debido a que existe el efecto de conversión desigual del capital cultural adquirido escolarmente. Para obtener tres categorías de respuesta la hemos recodificado. Esto se ha efectuado a partir de las categorías de ISCED-97 (OECD, 1999). Quedando categorizado en Obligatorios (niveles 1 y 2), Secundarios Post-obligatorios (3 y 4 ) y Terciarios (5 y 6). Y el índice seleccionado ha sido Recursos del hogar para el aprendizaje (Recu_Apre_scl). Como los dos anteriores, este ha sido creado a partir de varios ítems como, por ejemplo, mesa de estudio propia, libros infantiles en el hogar, ebooks para el niño o la niña etc... Los cuales son producto de la acumulación del capital económico y cultural, ya que además de ser una inversión económica, esta se hace expresamente en la formación académica de los descendientes.

Además, en los análisis previos a las regresiones logísticas binarias, hemos incluido otras variables. Dos variables dicotómicas: sexo e inmigrantes, por ser características sociodemográficas de 
relevancia. Y dos variables continuas: Ratio de alumnado por clase (Rat_alumn_cla) y Actividades tempranas de formación (Actv_Temprns). La primera para incluir una variable que esté relacionada con la inversión estatal (y privada) en la educación, siendo esta la única variable continua que no es un índice creado por la IEA, y por tanto no posee la misma escala. La segunda variable ha sido seleccionada con el propósito de incluir una cuestión comentada en el punto previo, los efectos del entorno son mayores a edades tempranas, como sostienen Carneiro y Heckman (2003). Esta segunda variable está construida a partir de la asistencia a escolar previa a la educación obligatoria, junto a otras variables que incluye el cuestionario del hogar y que son consideradas extracurriculares.

Respecto a la distribución de las variables politómicas (Tabla 2) nos encontramos con tres características que se repiten en los tres capitales que hemos seleccionado. En primer lugar, el estudiantado español proviene de familias con mayor capital. En segundo lugar, observamos que en las muestras marroquíes hay más casos perdidos en todas las variables. Esto, cuanto menos, es digno de señalar porque si existen problemas para encontrar investigaciones y estudios sobre la educación en el reino alauita, se necesitaría mayor calidad de estos estudios para compensar la poca cantidad que existe.

Por último, vemos que la distribución de las dos muestras marroquíes arroja cifras muy parejas en cada variable, lo que demuestra un buen trabajo de campo realizado, pues se sitúan dentro del error muestral. Estas tres conclusiones también son arrojadas por la tabla 3, la cual nos muestra las principales medidas descriptivas de los cuatro índices.

Respecto a las dos variables politómicas, sexo e inmigración, los números son bastante similares en las tres bases de datos. Destacando que en España el porcentaje de inmigrantes sea superior al de las dos bases de datos marroquíes por menos de un punto porcentual, esto puede deberse a dos cuestiones. La primera es que España posee más casos perdidos en esta variable (ASBH3A), además esta hace referencia a los nacidos fuera del país, independientemente del lugar de nacimiento de los padres y de su nacionalidad. Respecto al número de alumnos por clase ${ }^{12}$, España posee una media de casi siete alumnos menos.

Tabla 2. Distribución de las variables politómicas para cada una de las muestras.

\begin{tabular}{|c|c|c|c|c|c|c|c|}
\hline \multirow[t]{2}{*}{ Variables } & \multirow{2}{*}{$\begin{array}{l}\text { Categorías de } \\
\text { respuesta }\end{array}$} & \multicolumn{2}{|c|}{ Marruecos LP } & \multicolumn{2}{|c|}{ España PIRLS } & \multicolumn{2}{|c|}{ Marruecos PIRLS } \\
\hline & & Porcentaje & $\mathrm{N}$ & Porcentaje & $\mathrm{N}$ & Porcentaje & $\mathrm{N}$ \\
\hline \multirow[t]{5}{*}{$\begin{array}{l}\text { Ocupación más alta } \\
\text { de ambos padres }\end{array}$} & $\begin{array}{l}\text { Trabajadores } \\
\text { no cualificados }\end{array}$ & 21,4 & 775 & 5,6 & 545 & 21,6 & 792 \\
\hline & $\begin{array}{l}\text { Trabajadores } \\
\text { cualificados }\end{array}$ & 33,9 & 1226 & 33,3 & 3236 & 31,9 & 1167 \\
\hline & $\begin{array}{l}\text { Profesionales } \\
\text { y Empresarios }\end{array}$ & 16,7 & 605 & 45,6 & 4441 & 18,5 & 676 \\
\hline & Total & 72,0 & 2605 & 84,5 & 8222 & 72,0 & 2635 \\
\hline & No validos & 28,0 & 1012 & 15,5 & 1510 & 28,0 & 1023 \\
\hline \multirow{5}{*}{$\begin{array}{l}\text { Nivel de estudios } \\
\text { más alto de ambos } \\
\text { padres }\end{array}$} & Obligatorios & 49,3 & 1784 & 10,2 & 995 & 51,2 & 1873 \\
\hline & $\begin{array}{l}\text { Secundarios } \\
\text { post- } \\
\text { obligatorios }\end{array}$ & 23,1 & 837 & 42,1 & 4096 & 22,7 & 830 \\
\hline & Terciarios & 9,0 & 325 & 36,9 & 3587 & 8,5 & 313 \\
\hline & Total & 81,5 & 2946 & 89,2 & 8678 & 82,4 & 3016 \\
\hline & No validos & 18,5 & 671 & 10,8 & 1055 & 17,6 & 642 \\
\hline \multirow{4}{*}{$\begin{array}{l}\text { Número de libros en } \\
\text { el hogar }\end{array}$} & 0 a 10 & 54,3 & 1965 & 11,0 & 1066 & 55,2 & 2020 \\
\hline & 11 a 100 & 37,2 & 1344 & 59,8 & 5819 & 35,1 & 1285 \\
\hline & Más de 100 & 5,8 & 211 & 28,4 & 2765 & 6,3 & 231 \\
\hline & Total & 97,3 & 3520 & 99,2 & 9650 & 96,6 & 3536 \\
\hline
\end{tabular}

${ }^{12}$ Obsérvese que el producto de multiplicar la media de Rat_alumn_cla por el número de clases es mayor que el alumnado total, esto se debe a que se han incluido las clases del alumnado que no posee idoneidad de curso. 


\begin{tabular}{|l|l|l|l|l|l|l|l|}
\hline & No validos & 2,7 & 97 & 0,8 & 83 & 3,4 & 123 \\
\hline \multirow{4}{*}{ Inmigrante } & Autóctono & 89,7 & 3244 & 87,4 & 8511 & 88,7 & 3246 \\
\cline { 2 - 8 } & Inmigrante & 3,7 & 134 & 4,4 & 428 & 4,0 & 145 \\
\cline { 2 - 8 } & Total & 93,4 & 3378 & 91,8 & 8939 & 92,7 & 3391 \\
\cline { 2 - 8 } & No válidos & 6,6 & 239 & 8,2 & 794 & 7,3 & 267 \\
\hline \multirow{4}{*}{ Sexo } & Chicas & 48,0 & 1737 & 49,2 & 4791 & 47,6 & 1742 \\
\cline { 2 - 8 } & Chicos & 51,8 & 1875 & 50,6 & 4922 & 52,1 & 1906 \\
\cline { 2 - 8 } & Total & 99,9 & 3612 & 99,8 & 9712 & 99,7 & 3648 \\
\cline { 2 - 7 } & No válidos & 0,1 & 5 & 0,2 & 20 & 0,3 & 11 \\
\hline
\end{tabular}

Fuente: Elaboración propia a partir de datos de PIRLS 2016

Tabla 3. Valores Descriptivos de las cinco variables continuas en las tres muestras

\begin{tabular}{|l|c|c|c|c|c|c|c|c|c|c|c|c|}
\hline \multicolumn{1}{|c|}{ Variables } & \multicolumn{4}{|c|}{ Marruecos LP } & \multicolumn{4}{c|}{ España PIRLS } & \multicolumn{3}{c|}{ Marruecos PIRLS } \\
\hline & $\mathrm{N}$ & $\% \mathrm{CP}$ & $\mathrm{M}$ & $\mathrm{DT}$ & $\mathrm{N}$ & $\% \mathrm{CP}$ & $\mathrm{M}$ & $\mathrm{DT}$ & $\mathrm{N}$ & $\%$ CP & M & DT \\
\hline Recu_Apre_scl & 3081 & 14,8 & 7,04 & 2,21 & 8820 & 9,4 & 10,34 & 1,62 & 3121 & 14,7 & 7,07 & 2,25 \\
\hline Disp_Digt_scl & 3341 & 7,6 & 7,07 & 2,56 & 8894 & 8,6 & 10,09 & 1,73 & 3338 & 8,8 & 7,03 & 2,49 \\
\hline Gust_Lect_scl & 3365 & 7,0 & 8,95 & 2,09 & 8992 & 7,6 & 10,07 & 2,08 & 3365 & 8,0 & 8,97 & 2,03 \\
\hline Rat_alumn_cla & 362 & 6,2 & 30,20 & 10,12 & 674 & 0,6 & 23,77 & 6,37 & 362 & 6,2 & 30,2 & 10,11 \\
\hline Actv_Temprns & 3442 & 4,8 & 7,75 & 2,74 & 8993 & 7,6 & 10,65 & 1,89 & 3480 & 4,8 & 7,83 & 2,69 \\
\hline
\end{tabular}

Leyenda: \% CP indica el tanto por ciento de casos perdidos.

Fuente: Elaboración propia a partir de datos de PIRLS 2016

\subsection{El método de análisis}

Previo al modelo logit hemos realizado dos análisis bivariantes. Dos son las principales razones por las que los hemos realizado. La primera es para darle mayor robustez a los resultados. Y la segunda para poder testar variables que no pretendemos que incidan en el peso conjunto del modelo logit, ya sea por no ser variables propias de la familia, como el sexo del estudiantado, o por añadir una nueva dimensión, el caso de Actividades tempranas de formación, que añade la cuestión temporal, a diferencia de Recursos del hogar para el aprendizaje. Por ello, el análisis fundamental es el multivariante.

El primer análisis son unas tablas de contingencia para cada una de las muestras. En este, 10 son las variables explicativas: tres variables ordinales, dos dicotómicas y la reagrupación de las cinco variables numéricas.

En el siguiente análisis hemos utilizado la $R$ de Rosenthal a partir del test de Mann-Whitney, debido a que así podremos comparar variables ordinales y numéricas (Rosenthal, 1991; Rosnow y Rosenthal, 2003), y, por tanto, no tendremos que agrupar las variables numéricas. Además, únicamente Ratio de alumnado por clase posee una distribución normal, por lo que no podríamos haber realizado la $T$ de student. Ya que se han calculado los test de normalidad de KolmogorovSmirnov, y en los cuatro índices al cruzarse con $R P$ en todas las muestras el $p$-valor ha sido inferior a 0,01 . En este análisis no hemos podido incluir las variables sexo e inmigración por no ser ordinales.

El tercer análisis son tres Regresiones Logísticas Binarias. Una para cada muestra con las seis variables explicativas. Este análisis ha sido seleccionado por tres razones. La primera, nos permite contrastar las variables independientes politómicas y de intervalo con la variable dependiente, cuando esta última es dicotómica (Jovell, 1995; Pardo y Ruiz, 2013). La segunda, ya se ha utilizado 
para predecir el rendimiento escolar en España (Pérez et al., 2013; García-Fernández et al., 2013; Lozano y Trinidad, 2019). Por último, nos permite igualar el rendimiento de las evaluaciones de las tres bases de datos, al haber dicotomizado la variable dependiente. Este análisis nos permite comparar los diferentes capitales, la capacidad predictiva del conjunto de cada uno de los tres modelos, y, por consiguiente, comparar la incidencia del origen familiar en cada una de las muestras con mayor robustez que los anteriores análisis. Cabe decir, que estos análisis se han ajustado con un programa informático ${ }^{13}$.

Como nuestro objetivo es testar la influencia de los capitales económico y cultural de las familias no incluiremos en el análisis a las siguientes variables: sexo y la ratio de alumnado por clase, además esta última posee una escala diferente al no ser un índice generado por la IEA. Tampoco integraremos a la variable inmigración, además de ser dicotómica, y no tener tres categorías de respuesta, es poco predictora al concentrarse la mayoría de casos en una sola categoría. Por último, Actividades tempranas de formación es una variable continua que puede ser de interés, sin embargo, recursos del hogar para el aprendizaje ocupa el lugar de índice intermedio entre los capitales económico y cultural. Ya que está directamente relacionado con la familia y no con la intervención estatal, consideramos que sería falaz llegar a la conclusión de que el capital económico de la familia tiene mayor peso en un país en vías de desarrollo cuando entre las variables dependientes se encuentra alguna que mide la inversión estatal, y como hemos comentado la educación gratuita es más temprana en España. Además, no está en nuestro objetivo incluir entre las variables la dimensión temporal. Otra ventaja de haber incluido con anterioridad las variables ausentes en los análisis logit es comparar la distribución de estas con aquellas variables que sí incluimos en las regresiones.

Sin embargo, hemos percibimos que algunas variables suman una cantidad importante de datos perdidos. Concretamente en las dos muestras marroquíes, la variable Ocupación más alta de ambos padres posee un $28 \%$ y en Recursos del hogar para el aprendizaje más de un $14 \%$. La primera, como se ha comentado, no es sustituible por carencia de variables coincidentes para medir el capital económico en las muestras de ambos países y la segunda porque es el índice más adecuado para medir la acumulación del capital económico y el cultural. Además, las otras variables en mayor o menor medida suman casos perdidos, por lo que realizar un análisis multivariante conlleva que se acumulen los casos perdidos de diferentes variables en un mismo individuo, rebasando en ambas muestras marroquíes el $40 \%$, ver tabla 6 . Por lo que hemos optado por imputar los datos perdidos para el análisis de regresión logística binaria, que no para los análisis previos, ya que este análisis es el principal, pues los dos anteriores tienen como objetivo presentar las diferencias que explica la RLB. Debido a la cantidad de casos no validos (tabla 2) se ha realizado una Imputación múltiple de Little con cadenas de Markov, para más información véase el Anexo I.

\section{Resultados}

\subsection{Análisis bivariante con variables politómicas}

A continuación, nos vamos a centrar en las mayores diferencias de rendimiento según las variables seleccionadas para cada una de las muestras. En la tabla 4 se observa como a mayor capital familiar aumenta el rendimiento escolar. Respecto a Marr_LP, quienes tienen Padres con estudios universitarios poseen un 92,9\% de probabilidad de tener Alto Rendimiento (fila [f.] 20: columna[c.] 2 ), siendo la cifra más alta de toda la tabla. La segunda categoría que más casos concentra en $A R$ en esta base de datos es Profesionales y empresarios (80,1\%; f. 18: c. 2), muy por encima de aquellas variables que hemos catalogado dentro del capital cultural. La tercera mayor cifra, y última que rebasa el $75 \%$, es un nivel Alto para Recu_Apre_scl (f. 6: c. 2). Respecto al Bajo

\footnotetext{
${ }^{13}$ Este ha sido IBM SPSS Statics. Aunque solo se ha utilizado para el ajuste porque no calcula algunas operaciones, como la $R$ de Rosenthal o $R_{L}^{2}$.
} 
Rendimiento ninguna cifra acumula los tres cuartos de casos, aquella que más cerca está es el nivel Bajo para Actividades tempranas de formación con un 70,5\% (f. 13: c. 1), en la línea de Carneiro y Heckman (2003). Respecto a quienes han tenido un AR en Marruecos PIRLS, por encima del $75 \%$ se sitúan las dos mismas categorías y en el mismo orden que en la otra base de datos marroquí: Ambos padres con estudios universitarios (86,9\%; f. 21: c. 21) y Profesionales y empresarios (76,6\%; c. 8: f. 18), lo cual refuerza nuestra hipótesis y demuestra un buen trabajo de campo hecho por parte de la IEA. En BR también se repite en Marruecos PIRLS lo comentado en Marruecos LP: siendo las dos categoría Bajo la que más cantidad de individuos concentran en Actividades tempranas de formación (f. 13: c. 7) y en Recursos del hogar para el aprendizaje (f. 7: c. 4) con un $62,8 \%$. Por último, hemos de destacar que el Ratio de alumnado por clase no tenga valores significativos. Como hipótesis a esta cuestión, señalar la posibilidad de que las clases con menos alumnado se concentren en el entorno rural y que las familias de este, a su vez, posean menos capital que en los entornos urbanos.

En línea con estos resultados, y si se profundiza más, en la tabla 4 en Marruecos nos encontramos que las variables independientes que posteriormente incluiremos en la RLB tienen mayor peso que las restantes, con la salvedad de Actividades tempranas de formación, y dentro de las primeras las variables acumulativas son las de mayor preponderacia, en segundo lugar, aquellas propias del capital económico y, por último, las que manifiestan el capital cultural.

Respecto a la muestra española, las variables que más casos concentran en Bajo Rendimiento son las tres politómicas que miden el capital de la familia, siendo estas las únicas que superan el $75 \%$ en quienes poseen el menor de los capitales. Aun así, reforzando nuestra hipótesis de que el capital cultural tiene mayor peso en España que en Marruecos, su orden de mayor a menor es Libr_Hoga (82,7\%; f. 22: c. 4), Estud_PyM (80,3\%; f. 19: c. 4) y Ocup_PyM (75,8; c. 4: f. 16). La concentración de casos en Alto Rendimiento es menor, obteniendo la probabilidad más alta en quienes poseen un alto capital en Recursos del hogar para el aprendizaje con un 74,9\% (f.6: c. 5). Respecto a las variables que no incluiremos en el análisis de RLB encontramos dos diferencias con Marruecos. La primera es que las variables Rat_alumn_cla y Actv_Temprns poseen cifras similares $y$, la segunda, el mayor rendimiento de las chicas respecto a los chicos es menos agudo en España.

Tabla 4. Rendimiento en las tres pruebas PIRLS según cada una de las diez variables politómicas e independientes. Porcentaje en horizontal

\begin{tabular}{|c|c|c|c|c|c|c|c|c|c|c|}
\hline \multirow[t]{3}{*}{ Variable Explicativa } & \multirow{3}{*}{$\begin{array}{l}\text { Categorías de } \\
\text { respuesta }\end{array}$} & \multicolumn{3}{|c|}{ Marruecos LP } & \multicolumn{3}{|c|}{ España PIRLS } & \multicolumn{3}{|c|}{ Marruecos PIRLS } \\
\hline & & \multicolumn{3}{|c|}{ Rendimiento Prueba } & \multicolumn{3}{|c|}{ Rendimiento Prueba } & \multicolumn{3}{|c|}{ Rendimiento Prueba } \\
\hline & & Bajo & Alto & $\mathrm{N}$ & Bajo & Alto & $\mathrm{N}$ & Bajo & Alto & $\mathrm{N}$ \\
\hline \multirow{3}{*}{$\begin{array}{l}\text { Gusto por la lectura } \\
\text { de ambos padres }\end{array}$} & Bajo & 67,6 & 32,4 & 1079 & 63,4 & 36,6 & 3024 & 61,8 & 38,2 & 1174 \\
\hline & Medio & 45,7 & 54,3 & 1060 & 46,2 & 53,8 & 2832 & 49,9 & 50,1 & 991 \\
\hline & Alto & 31,8 & 68,2 & 1226 & 33,9 & 66,1 & 3137 & 33,5 & 66,5 & 1200 \\
\hline \multirow{3}{*}{$\begin{array}{l}\text { Recursos del hogar } \\
\text { para el aprendizaje }\end{array}$} & Bajo & 65,5 & 34,5 & 1126 & 74,1 & 25,9 & 2409 & 62,8 & 37,2 & 812 \\
\hline & Medio & 51,6 & 48,4 & 746 & 51,2 & 48,8 & 2942 & 53,2 & 46,8 & 1081 \\
\hline & Alto & 21,8 & 78,2 & 1209 & 25,1 & 74,9 & 3470 & 28,8 & 71,2 & 1228 \\
\hline \multirow{3}{*}{$\begin{array}{l}\text { Dispositivos digitales } \\
\text { en el hogar }\end{array}$} & Bajo & 65,9 & 34,1 & 1507 & 62,3 & 37,7 & 2705 & 62,4 & 37,6 & 1528 \\
\hline & Medio & 52,3 & 47,7 & 411 & 51,7 & 48,3 & 2121 & 58,6 & 41,4 & 370 \\
\hline & Alto & 25,6 & 74,4 & 1423 & 35,8 & 64,2 & 4069 & 27,8 & 72,2 & 1440 \\
\hline \multirow{3}{*}{$\begin{array}{l}\text { Ratio de alumnado } \\
\text { por clase }\end{array}$} & Bajo & 47,3 & 52,7 & 91 & 64,2 & 35,8 & 148 & 42,1 & 57,9 & 95 \\
\hline & Medio & 49,3 & 50,7 & 75 & 53,1 & 46,9 & 81 & 50,7 & 49,3 & 73 \\
\hline & Alto & 53,1 & 46,9 & 64 & 37,2 & 62,8 & 188 & 58,7 & 41,3 & 63 \\
\hline Actividades & Bajo & 70,5 & 29,5 & 1025 & 64,2 & 35,8 & 2538 & 62,8 & 37,2 & 1135 \\
\hline
\end{tabular}




\begin{tabular}{|c|c|c|c|c|c|c|c|c|c|c|}
\hline \multirow{2}{*}{$\begin{array}{l}\text { tempranas } \\
\text { formación }\end{array}$} & Medio & 45,9 & 54,1 & 1215 & 50,3 & 49,7 & 3027 & 47,6 & 52,4 & 1140 \\
\hline & Alto & 35,2 & 64,8 & 1271 & 33,9 & 66,1 & 3476 & 36,6 & 63,4 & 1204 \\
\hline \multirow[t]{3}{*}{$\begin{array}{l}\text { Ocupación más alta } \\
\text { de ambos padres }\end{array}$} & $\begin{array}{l}\text { Trabajadores } \\
\text { no } \\
\text { cualificados }\end{array}$ & 60,9 & 39,1 & 774 & 75,8 & 24,2 & 545 & 60,6 & 39,4 & 792 \\
\hline & $\begin{array}{l}\text { Trabajadores } \\
\text { cualificados }\end{array}$ & 44 & 56 & 1226 & 56 & 44 & 3236 & 48,9 & 51,1 & 1167 \\
\hline & $\begin{array}{l}\text { Profesionales } \\
\text { y empresarios }\end{array}$ & 19,9 & 80,1 & 604 & 34,1 & 65,9 & 4440 & 23,4 & 76,6 & 676 \\
\hline \multirow{3}{*}{$\begin{array}{l}\text { Nivel de estudios } \\
\text { más alto de ambos } \\
\text { padres }\end{array}$} & Obligatorios & 61,5 & 38,5 & 1784 & 80,3 & 19,7 & 995 & 58,9 & 41,1 & 1874 \\
\hline & $\begin{array}{l}\text { Secundarios } \\
\text { post- } \\
\text { obligatorios }\end{array}$ & 27,1 & 72,9 & 838 & 55 & 45 & 4096 & 29,3 & 70,7 & 829 \\
\hline & Terciarios & 7,1 & 92,9 & 325 & 27,9 & 72,1 & 3587 & 13,1 & 86,9 & 313 \\
\hline \multirow{3}{*}{$\begin{array}{l}\text { Número de libros en } \\
\text { el hogar }\end{array}$} & 0 a 10 & 62,7 & 37,3 & 1965 & 82,7 & 17,3 & 1066 & 61,1 & 38,9 & 2020 \\
\hline & 11 a 100 & 32,1 & 67,9 & 1344 & 50,7 & 49,3 & 5819 & 34,7 & 65,3 & 1284 \\
\hline & Más de 100 & 35,5 & 64,5 & 211 & 34,6 & 65,4 & 2766 & 30,7 & 69,3 & 231 \\
\hline \multirow[t]{2}{*}{ Sexo } & Chicas & 40,4 & 59,6 & 1742 & 47,1 & 52,9 & 4796 & 42,3 & 57,7 & 1829 \\
\hline & Chicos & 59,1 & 40,9 & 1874 & 52,9 & 47,1 & 4937 & 57,1 & 42,9 & 1829 \\
\hline \multirow[t]{2}{*}{ Inmigrante } & Inmigrante & 67,9 & 32,1 & 134 & 66,0 & 34,0 & 429 & 59,6 & 40,4 & 146 \\
\hline & Autóctono & 47,1 & 52,9 & 3244 & 46,6 & 53,4 & 8511 & 47,3 & 52,7 & 3246 \\
\hline
\end{tabular}

En las 18 tablas de contingencia que la componen la significación de chi-cuadrado de Pearson es: $p<0,01$. Salvo para las dos muestras marroquíes en la variable Ratio de alumnado por clase, donde $p$ es 0,119 para Marruecos PIRLS y 0,771 para Marruecos LP. Fuente: Elaboración propia a partir de datos de PIRLS 2016.

\subsection{Análisis bivariante a partir de rangos}

En el análisis bivariante de la Tabla 5, se muestra que los resultados para Ratio de alumnado por clase no son estadísticamente significativos, en esta ocasión también en el caso español. En conjunto, la $R$ de Rosenthal obtiene valores moderados. Respecto a la metodología, en esta ocasión, hemos podido respetar la forma en la que la IEA genera sus índices. Cabe destacar, que el signo negativo se debe a que el 0 representa el Bajo Rendimiento y el 1 el Alto rendimiento, para seguir con la tradición en los modelos de Regresión Logística Binaria (Jovell, 1995), análisis que se hará posteriormente.

Si hacemos un análisis por nivel de medida y en cada base de datos los resultados son los que exponemos a continuación.

Respecto a los índices en Marruecos_LP son, de mayor a menor, Recursos del hogar para el aprendizaje (-0,3979; f. 2: c. 3), Dispositivos digitales en el hogar (-0,3769; f. 3: c. 3) y Gusto por la lectura de ambos padres (-0,2886; f. 1: c. 3). Para Marruecos PIRLS Dip_Digt_scl (-0,3428; f. 3: c. 9), Recu_Apre_scl (-0,3161; f. 2; c. 9) y Gust_Lect_scl $(-0,2491 ;$ f. 1: c. 9). En ambas muestras se observa que la variable seleccionada para medir el capital cultural es aquella que menor poder explicativo tiene en Rendimiento Prueba en Marruecos. Como pronosticaba nuestra hipótesis en el caso español el capital cultural tiene mayor influencia que el capital económico debido a la perdida de capacidad explicativa de este último, siendo el consiguiente orden: Recu_Apre_scl (0,4138; f. 2: c. 6), Gust_Lect_scl (-0,2492; f. 1: c. 6), y Dip_Digt_scl (-0,2421; f. 3: c. 6).

Respecto a las variables ordinales de estos mismos capitales el orden, de mayor a menor influencia en $R P$, es para las tres bases de datos: Estud_PyM,Ocup_PyM y Libr_Hoga. Lo cual, a priori, contradice nuestra hipótesis, pues el capital económico (Ocup_PyM: -0,2486; f. 6: c. 6) se sitúa en España por encima del capital cultural (Libr_Hoga: -0,2468; f. 5: c. 6). Sin embargo, la diferencia entre ambas $R$ de Rosenthal es menor de 0,002 , por lo que encontrado una excepción esta es muy pequeña, pudiendo afirmar que en las variables ordinales en España el capital económico es mucho más similar que en Marruecos, donde las $R$ de Rosenthal de ambas variables tienen diferencias mayores de 0,008. 
Respecto a las dos variables que son de contexto encontramos resultados dispares. Actividades tempranas de formación en Marruecos posee valores moderados, situándose en ambas bases de datos con valores más bajos que los otros tres índices de la IEA anteriormente analizados. Sin embargo, en España es el segundo índice con un valor más alto, con -0,2686 (f. 6: c. 6). Por último, Ratio de alumnado por clase vuelve a no ser significativa en las dos bases marroquíes, pero esta vez tampoco lo es para el caso español.

En resumen, los resultados que arroja la tabla 5 son concordantes con las tablas de contingencia con dos excepciones. La primera es que para el caso español Dip_Digt_scl tiene mayor peso que Gust_Lect_scl, si bien la diferencia entre ambas variables es menor que en las muestras marroquíes. La segunda es que las variables que hemos denominado acumulativas, pues reflejan los capitales cultural y económico, vuelven a ser las que más peso tienen con una excepción: en Marruecos_PILS Dispositivos digitales en el hogar es la variable independiente con mayor capacidad de todas, lo cual refuerza nuestra hipótesis.

Tabla 5. $U$ de Mann-Whitney y $R$ de Rosenthal de las cinco variables numéricas y las tres variables ordinales según el rendimiento en las pruebas PIRLS

\begin{tabular}{|c|c|c|c|c|c|c|c|c|c|}
\hline \multirow[t]{2}{*}{ Variables independientes } & \multicolumn{3}{|c|}{ Marruecos LP } & \multicolumn{3}{|c|}{ España PIRLS } & \multicolumn{3}{|c|}{ Marruecos PIRLS } \\
\hline & $u$ & $\mathrm{p}$ & $\mathrm{R}$ & $u$ & $\mathrm{p}$ & $\mathrm{R}$ & $U$ & $\mathrm{p}$ & $\mathrm{R}$ \\
\hline $\begin{array}{c}\text { Gusto por la lectura de ambos } \\
\text { padres }\end{array}$ & 1084225 & 0,00 & $-0,2886$ & 6518639 & 0,00 & $-0,2492$ & 1111676 & 0,00 & $-0,2491$ \\
\hline $\begin{array}{r}\text { Recursos del he } \\
\text { aprendi }\end{array}$ & 4437 & 0,00 & 79 & 4597124 & 0,00 & $-0,4138$ & 853494 & 0,00 & $-0,3161$ \\
\hline Dispositivos digitales en el hogar & 914499 & 0,00 & $-0,3769$ & 6506738 & 0,00 & $-0,2421$ & 943619 & 0,00 & $-0,3428$ \\
\hline Ratio de alumn & 6567 & 0,93 & $-0,0059$ & 19996 & 0,18 & $-0,0652$ & 6013 & 0,20 & $-0,0851$ \\
\hline $\begin{array}{r}\text { Actividades te } \\
\text { forma }\end{array}$ & 1162838 & 0,00 & $-0,2765$ & 6325496 & 0,00 & $-0,2686$ & 1284663 & 0,00 & $-0,2054$ \\
\hline $\begin{array}{c}\text { Ocupación más alta de ambos } \\
\text { padres }\end{array}$ & 670822 & 0,00 & $-0,2854$ & 5645562 & 0,00 & $-0,2486$ & 681053 & 0,00 & $-0,2669$ \\
\hline $\begin{array}{l}\text { Nivel de estudios más alto de } \\
\text { ambos padres }\end{array}$ & 02 & 0,00 & $-0,4$ & 5473386 & 0,00 & $-0,3389$ & 826159 & 0,00 & $-0,3415$ \\
\hline Número de libros en el hogar & 1288494 & 0,00 & $-0,2759$ & 7939420 & 0,00 & $-0,2468$ & 1287674 & 0,00 & $-0,2576$ \\
\hline
\end{tabular}

Fuente: Elaboración propia a partir de datos de PIRLS 2016

\subsection{Análisis Multivariante: Regresión Logística Binaria}

En los dos métodos bivariantes hemos comprobado que en España el capital cultural de las familias posee mayor concentración de casos en las categorías no intermedias que el capital económico de estas, mientras en Marruecos el económico posee mayor preponderancia. Si bien, en las tablas de contingencia los resultados eran inequívocos, en la $R$ de Rosenthal no ocurría lo mismo con las variables ordinales en España PIRLS, aunque en este caso las diferencias eran muy leves. Por esto último, el modelo logit, con mayor capacidad explicativa, al ser un análisis multivariante, y con los casos perdidos imputados, nos proporciona el principal análisis para poder testar nuestra hipótesis. 
Los modelos ajustados son tres (Tabla 6), uno para cada base de datos. Marruecos LP tiene tanto la mayor capacidad predictiva como el ajuste, seguido de España PIRLS y, por último, Marruecos PIRLS. Pudiendo afirmar que los modelos son cuanto menos moderados.

Tabla 6. Ajuste de los tres modelos de RLB

\begin{tabular}{|l|l|l|l|l|l|l|l|l|l|}
\hline Modelo & $\begin{array}{l}\text { Casos } \\
\text { válidos }\end{array}$ & $\begin{array}{l}\% \text { casos } \\
\text { recuperados }\end{array}$ & $G_{0-1}^{2}$ & $\mathrm{p}$ & $\begin{array}{l}\% \text { valor } \\
\text { predictivo }\end{array}$ & $\begin{array}{l}(-) 2 \quad \text { Log } \\
\text { likelihood }\end{array}$ & $\mathrm{R}^{2}$ & $\mathrm{R}_{\text {ajustado }}^{2}$ & $\mathrm{R}_{\mathrm{L}}^{2}$ \\
\hline Marruecos LP & 13,2 & 40,5 & 25435,9 & 0,000 & 71,6 & 99917,01 & 0,245 & 0,327 & 0,203 \\
\hline España PIRLS & 9,5 & 16,3 & 57797,8 & 0,000 & 70,4 & 279516,72 & 0,211 & 0,282 & 0,171 \\
\hline $\begin{array}{l}\text { Marruecos } \\
\text { PIRLS }\end{array}$ & 12,5 & 42,0 & 19530,2 & 0,000 & 69,7 & 107254,70 & 0,192 & 0,256 & 0,154 \\
\hline
\end{tabular}

El R ${ }^{2}$ realizado es el de Cox y Snell y el $R_{\text {ajustado }}^{2}$ el de Nagelkerke (172-176, Pardo y Ruiz, 2013).

Fuente: Elaboración propia a partir de datos de PIRLS 2016.

La tabla 7 nos muestra el modelo RLB Marruecos $L P$, siendo logit (Alto Rendimiento $=1)=-3,387+$ 0,136 (Gust_Lect_scl) + 0,030 (Recu_Apre_scl) +0,147 (Disp_digt_scl) + 0,503 (Ocup_PyM $=$ Cualificados) $+0,877$ (Ocup_PyM $=$ Profesionales y empresarios) $+0,663$ (Estud_PyM $=$ Secundarios post-obligatorios $)+1,879$ (Estud_PyM $=$ Universitarios $)+0,546($ Libr_hoga $=11$ a 100 $)+-0,033$ ( $L i b r \_h o g a=$ más de 100), ya que $B$ representa el coeficiente de regresión asociado a la variable independiente, siendo el logaritmo de las odds, salvo para la constante que es el valor que adquiere esta.

Respecto a los índices generados por el propio estudio, las odds ratio (OR) muestran, en línea con los análisis previos, que el capital económico tiene mayor capacidad predictiva que aquellas variables seleccionadas para el capital cultural en Marruecos. Exactamente igual ocurre con las cuestiones seleccionadas del cuestionario del hogar, donde hay una categoría que no es suficientemente significativa: Libr_hoga = más de 100 (f. 9: c. 5). Pues su p-valor del índice de Wald es mayor de 0,05 , lo cual refuerza tanto el marco teórico como los resultados hasta aquí expuestos. Como excepción de los resultados arrojados anteriormente, cabe destacar, que la variable que acumula a ambos capitales (Recu_Apre_scl) es la que menor odds ratio posee dentro de los índices (f. 2: c. 6). En contraposición, la variable ordinal que concentra ambos capitales en Estud_PyM = Universitarios aumenta 6,548 odds (f. 7: c. 6) respecto a Estud_PyM = Obligatorios, siempre que el resto de las variables permanezcan constantes.

El modelo RLB Marruecos PIRLS (tabla 8) queda: logit (Alto Rendimiento $=1)=-2,358+0,120$ (Gust_Lect_scl) + -0,122 (Recu_Apre_scl) + 0,178 (Disp_Digt_scl) + 0,285 (Ocup_PyM = Cualificados) $+0,909$ (Ocup_PyM $=$ Profesionales y empresarios) $+0,749$ (Estud_PyM $=$ Secundarios post-obligatorios $)+1,496$ (Estud_PyM $=$ Universitarios $)+0,618($ Libr_hoga $=11$ a 100 $)+0,418$ (Libr_hoga = más de 100). 
LOZANO PÉREZ y TRINIDAD REQUENA, La capacidad predictiva en el rendimiento escolar ...

Tabla 7. Resultados del modelo para Marruecos LP

\begin{tabular}{|c|c|c|c|l|l|l|l|l|}
\hline & $\mathrm{B}$ & $\mathrm{EE}$ & Wald & $\mathrm{Gl}$ & $\mathrm{p}$ & $\mathrm{OR}$ & \multicolumn{2}{|c|}{$95 \% \mathrm{I} \mathrm{OR}_{\mathrm{O}}$} \\
\hline Gust_Lect_scl & 0,136 & 0,004 & 1038,07 & 1 & 0,000 & 1,145 & 1,136 & 1,155 \\
\hline Recu_Apre_scl & 0,030 & 0,006 & 21,63 & 1 & 0,000 & 1,030 & 1,017 & 1,043 \\
\hline Disp_Digt_scl & 0,147 & 0,004 & 1403,06 & 1 & 0,000 & 1,159 & 1,150 & 1,168 \\
\hline Ocup_PyM = Cualificados & 0,503 & 0,018 & 817,73 & 1 & 0,000 & 1,654 & 1,598 & 1,712 \\
\hline $\begin{array}{c}\text { Ocup_PyM = Profesionales y } \\
\text { empresarios }\end{array}$ & 0,877 & 0,025 & 1223,95 & 1 & 0,000 & 2,404 & 2,288 & 2,525 \\
\hline $\begin{array}{c}\text { Estud_PyM = Secundarios post- } \\
\text { obligatorios }\end{array}$ & 0,663 & 0,020 & 1107,18 & 1 & 0,000 & 1,941 & 1,867 & 2,019 \\
\hline Estud_PyM = Universitarios & 1,879 & 0,043 & 1899,17 & 1 & 0,000 & 6,548 & 6,017 & 7,125 \\
\hline Libr_Hoga = 11 a 100 & 0,546 & 0,019 & 843,30 & 1 & 0,000 & 1,726 & 1,663 & 1,790 \\
\hline Libr_Hoga = más de 100 & $-0,033$ & 0,037 & 0,78 & 1 & 0,377 & 0,968 & 0,899 & 1,041 \\
\hline Constante & $-3,387$ & 0,046 & 5493,11 & 1 & 0,000 & 0,034 & & \\
\hline
\end{tabular}

Fuente: Elaboración propia a partir de datos de PIRLS 2016

Tabla 8. Resultados del modelo para Marruecos PIRLS

\begin{tabular}{|c|c|c|c|c|c|c|c|c|}
\hline & $\mathrm{B}$ & $\mathrm{EE}$ & Wald & $\mathrm{Gl}$ & $\mathrm{p}$ & $\mathrm{OR}$ & \multicolumn{2}{|c|}{$95 \% \mathrm{IC}$} \\
\hline Gust_Lect_scl & 0,120 & 0,004 & 850,24 & 1 & 0,000 & 1,127 & 1,118 & 1,136 \\
\hline Recu_Apre_scl & $-0,122$ & 0,006 & 389,71 & 1 & 0,000 & 0,885 & 0,875 & 0,896 \\
\hline Disp_Digt_scl & 0,178 & 0,004 & 2132,91 & 1 & 0,000 & 1,194 & 1,185 & 1,203 \\
\hline Ocup_PyM = Cualificados & 0,285 & 0,017 & 277,90 & 1 & 0,000 & 1,330 & 1,286 & 1,375 \\
\hline $\begin{array}{c}\text { Ocup_PyM = Profesionales y } \\
\text { empresarios }\end{array}$ & 0,909 & 0,023 & 1519,84 & 1 & 0,000 & 2,483 & 2,372 & 2,599 \\
\hline $\begin{array}{c}\text { Estud_PyM = Secundarios } \\
\text { post-obligatorios }\end{array}$ & 0,749 & 0,020 & 1418,95 & 1 & 0,000 & 2,114 & 2,033 & 2,198 \\
\hline Estud_PyM = Universitarios & 1,496 & 0,037 & 1646,18 & 1 & 0,000 & 4,465 & 4,154 & 4,800 \\
\hline Libr_Hoga = 11 a 100 & 0,618 & 0,018 & 1120,52 & 1 & 0,000 & 1,855 & 1,789 & 1,923 \\
\hline Libr_Hoga = más de 100 & 0,418 & 0,037 & 125,88 & 1 & 0,000 & 1,519 & 1,412 & 1,634 \\
\hline Constante & $-2,358$ & 0,044 & 2874,07 & 1 & 0,000 & 0,095 & & \\
\hline
\end{tabular}

Fuente: Elaboración propia a partir de datos de PIRLS 2016

El p-valor del test de Wald (c. 5) muestra como todos sus valores son significativos, véase tabla 8. Una vez más, las odds obtenidas nos muestran que en Marruecos las variables económicas tienen más peso que aquellas que miden el capital cultural, pues el OR de Disp_Digt_scl (f. 3: c. 6) es mayor que el que posee Gust_Lect_scl (f. 1: c. 6), y los que adquiere Ocup_PyM son mayores que aquellos que posee Libr_Hoga. Destacando que quien posee al padre o la madre en la categoría Profesionales y empresarios tiene un $183 \%$ más de posibilidades de tener alto rendimiento que quien tiene progenitores con ocupaciones sin cualificación (f. 5: c. 6) permaneciendo constantes el resto de las variables. Además, el OR de Libr_Hoga $=11$ a 100 (f. 8: c. 6) es mayor que el valor de Libr_Hoga = más de 100 (f. 9: c. 6), lo que refuerza nuestra hipótesis. Por último, destacar que las variables que hemos denominado intermedias tienen valores dispares, su índice (Recu_Apre_scl; f.2: c. 6) posee un OR muy bajo. En contraposición, su variable ordinal (Estud_PyM) tiene los valores más altos, siendo 4,465 el OR (f. 7: c. 6) para quienes pertenecen a la categoría Estud_PyM 
= Universitarios respecto a quienes se sitúan en Estud_PyM = Obligatorios permaneciendo constantes el resto de variables.

Hasta aquí hemos examinado las dos regresiones de las dos muestras marroquíes, ahora nos centraremos en el caso español (Tabla 9). Siendo logit (Alto Rendimiento $=1)=-8,145+0,085$ (Gust_lect_scl) + 0,392 (Recu_Apre_scl) $+0,151$ (Disp_digt_scl) $+0,369$ (Ocup_PyM $=$ Cualificados) $+0,341$ (Ocup_PyM $=$ Profesionales y empresarios) $+0,491$ (Estud_PyM $=$ Secundarios postobligatorios) $+0,875$ (Estud_PyM $=$ Universitarios $)+0,927($ Libr_hoga $=11$ a 100) $+0,844$ (Libr_hoga = más de 100).

En las variables ordinales los resultados muestran como el capital cultural tiene mayor capacidad para predecir el Alto Rendimiento que el capital económico. Ya que Ocup_PyM (c. 6) posee valores más bajos en las OR que Libr_Hoga (c. 6), la cual supera a Estud_PyM (c. 6). En este punto, destacar que, con referencia a Ocup_PyM $=$ No cualificados, Ocup_PyM $=$ Cualificados posee 1,447 OR (f. 4: c. 6) siendo mayor que la de Ocup_PyM = Profesionales y empresarios $(1,406 ;$ f. 5 : c. 6). Sin embargo, en los índices, Gust_Lect_scl (f. 1: c. 6) tiene un valor menor que Disp_Digt_scl (f. 1: c. 6), al igual que ocurría en la $R$ de Rosenthal.

Tabla 9. Resultados del modelo para España PIRLS

\begin{tabular}{|l|l|l|l|l|l|l|l|l|}
\hline & $\mathrm{B}$ & $\mathrm{EE}$ & Wald & $\mathrm{Gl}$ & $\mathrm{p}$ & \multicolumn{2}{l|}{ OR } & \multicolumn{2}{|c|}{$95 \%$ IC $\mathrm{OR}$} \\
\hline Gust_Lect_scl & 0,085 & 0,002 & 1232,86 & 1 & 0,000 & 1,089 & 1,083 & 1,094 \\
\hline Recu_Apre_scl & 0,392 & 0,006 & 4526,91 & 1 & 0,000 & 1,479 & 1,462 & 1,496 \\
\hline Disp_Digt_scl & 0,151 & 0,003 & 2588,53 & 1 & 0,000 & 1,162 & 1,156 & 1,169 \\
\hline Ocup_PyM = Cualificados & 0,369 & 0,021 & 304,10 & 1 & 0,000 & 1,447 & 1,388 & 1,508 \\
\hline $\begin{array}{l}\text { Ocup_PyM = Profesionales } \\
\text { y empresarios }\end{array}$ & 0,341 & 0,022 & 233,02 & 1 & 0,000 & 1,406 & 1,346 & 1,469 \\
\hline $\begin{array}{l}\text { Estud_PyM = Secundarios } \\
\text { post-obligatorios }\end{array}$ & 0,491 & 0,018 & 747,32 & 1 & 0,000 & 1,634 & 1,577 & 1,692 \\
\hline $\begin{array}{l}\text { Estud_PyM } \\
\text { Universitarios }\end{array}$ & 0,875 & 0,021 & 1729,94 & 1 & 0,000 & 2,400 & 2,303 & 2,501 \\
\hline Libr_Hoga = 11 a 100 & 0,927 & 0,018 & 2525,76 & 1 & 0,000 & 2,528 & 2,438 & 2,621 \\
\hline Libr_Hoga = más de 100 & 0,844 & 0,021 & 1541,82 & 1 & 0,000 & 2,325 & 2,229 & 2,425 \\
\hline Constante & $-8,145$ & 0,056 & 20871,12 & 1 & 0,000 & 0,000 & & \\
\hline
\end{tabular}

Fuente: Elaboración propia a partir de datos de PIRLS 2016

Por último, para obtener una imagen más global, en el análisis de las tres regresiones logísticas binarias se observa que el capital económico de las familias tiene más peso que el capital cultural en Marruecos. Si bien, es cierto que los resultados en Marruecos son unánimes, en el caso español encontramos resultados contradictorios, pues las variables politómicas, que utilizaba el propio Bourdieu en solitario (1979; 2011) y junto con Passeron (1964), refuerzan de forma notable nuestra hipótesis. Sin embargo, los índices utilizados por la IEA no refuerzan nuestra hipótesis, aunque los resultados son más homogéneos que en las variables politómicas. Para el caso español, la cuestión de que los índices que se generan para medir el capital cultural sean menos preponderantes que las variables utilizadas por Bourdieu también ocurre en PISA (Lozano y Trinidad, 2019).

\section{Conclusiones}

Nuestras hipótesis son que el capital cultural de las familias tiene mayor preponderancia en el rendimiento académico en España, por contraposición, en Marruecos el capital económico posee mayor capacidad predictiva. Para ello hemos utilizado los datos de PIRLS 2016 con tres bases de datos: dos marroquíes y una española. Habiendo realizado tres análisis estadísticos diferentes: Tablas de contingencia, análisis bivariante de rangos y regresiones logísticas, estás últimas con valores imputados por Imputación Múltiple siendo el principal análisis. 
Como hemos utilizado dos tipos de variables, índices de la propia IEA y variables ordinales, en total se ha comparado la influencia del capital cultural con el económico en el rendimiento de la prueba PIRLS 201612 veces en Marruecos y seis en España. Las 12 veces que se ha efectuado en Marruecos los resultados han sido unánimes: el capital económico, respecto al cultural, de las familias posee valores más preponderantes para el rendimiento académico. En el caso español nuestra hipótesis también ha sido confirmada, sin embargo, estos resultados hay que interpretarlos con mayor cautela, pues el capital cultural ha poseído mayor influencia que el económico en cuatro de las seis comparaciones, siendo cierto que en las dos excepciones el margen es muy estrecho.

Por último, hay una serie de cuestiones metodológicas que cabe señalar. La principal es que los análisis de regresión logística multivariante, junto con la imputación múltiple, han confirmado los resultados de los análisis bivariantes. Respecto a la base de datos de la IEA, señalar que en la mayoría de los análisis las variables que atienden a cuestiones directas del cuestionario se han comportado con mayor capacidad predictiva que los índices generados por la propia IEA, siendo la excepción el análisis bivariante de rangos. Y que los campos de la sociología de la educación y de la economía de la educación requieren de mayor cantidad de estudios en países en vías de desarrollo por parte de las evaluaciones internacionales, así como mayor calidad de estos.

\section{Referencias}

ALLISON, Paul D. (2001): Missing data, Thousand Oaks, Series on Quantitative Application in the social sciences, $\mathrm{n}$ ㅇ 136, Sage Publications.

ÁLVAREZ-SOTOMAYOR, Alberto (2012): "Educación y capital social", en TRINIDAD, Antonio y GÓMEZ, Francisco J. (Coords.): Sociedad, familia, educación: Una introducción a la sociología de la educación, Madrid, Tecnos, pp. 229-240.

ÁLVAREZ-SOTOMAYOR, Alberto y MARTÍNEZ-COUSINOU, Gloria (octubre de 2016) “¿Capital económico o cultural? El efecto del origen social sobre las desventajas académicas de los hijos de inmigrantes en España" Papers, vol. 101, no 4, pp. 527-554. http://dx.doi.org/10.5565/rev/papers.2200

BIJOU, Mohammed y BENNOUNA, Narjis: "Dépenses publiques éducatives et performance scolaire au Maroc. Une analyse multiniveaux à partir des données TIMSS 2015", HAL, 20 de enero de 2018, disponible en https://hal.archives-ouvertes.fr/hal-01689120 [consulta: 12 de junio de 2019]

BOUDON, Raymond (1974): Education, Opportunity and Social Inequality, Nueva York, WileyInterscience.

BOURDIEU, Pierre (1979): La distinction: critique sociale du jugement, Paris, Les éditions de Minuit. BOURDIEU, Pierre (2011): Capital cultural, escuela y espacio social, México, Siglo XXI.

BOURDIEU, Pierre y PASSERON, Jean-Claude (1964): Les héritiers: les étudiants et la culture, Paris, Les éditions de Minuit.

CARNEIRO, Pedro y HECKMAN, James J. (2003): "Human capital policy", en HECKMAN, James J. y KRUEGER, Alan B. (Eds.): Inequality in America: What Role for Human Capital Policies?, Cambridge, MIT Press, pp. 77-239.

COLEMAN, James S. (abril de 1968): "The concept of equality of educational opportunity", Harvard Educational Review, no 38, pp. 7-22. https://doi.org/10.17763/haer.38.1.m3770776577415m2 
DE GRAFF, Nan Dirk; DE GRAFF, Paul M. y KRAAYKAMP, Gerbert (abril de 2000): "Parental Cultural Capital and Educational Attainment in the Netherlands: A Refinement of the Cultural Capital Perspective", Sociology of Education, no 73, vol. 2, pp. 92-111. http://dx.doi.org/10.2307/2673239>

ENDERS, Craig K. (2010): Applied Missing Data Analysis-Methodology in Social Sciences, New York, Guilford Press.

FASFOUS, Ahmed F; HIDALGO-RUZZANTE, Natalia; VILAR-LÓPEZ, Raquel; CATENA-

MARTINEZ, Andrés y PÉREZ-GARCÍA, Miguel (diciembre de 2013): "Cultural differences in neuropsychological abilities required to perform intelligence tasks", Archives of clinical neuropsychology, vol. 28, no8, pp. 784-790. https://doi.org/10.1093/arclin/act074

FERNÁNDEZ, María (2015): “Continuidad o cambio en la desigualdad de oportunidades educativas: evidencia internacional y teorías", Revista Española de Sociología, no 23, pp. 151-164.

FOY, Pierre (2018): PIRLS 2016: User Guide for the International Database (IEA), International Association for the Evaluation of Educational Achievement, disponible en https://timssandpirls.bc.edu/pirls2016/international-database/downloads/P16 UserGuide.pdf [consulta: 30 de mayo de 2019].

GARCÍA, José (2013): "Socioeconomic Level, Type of School and Educational Results in Spain: the Case for Spain - TIMSS PIRLS 2011", en Instituto Nacional de Evaluación Educativa (Ed.): PIRLS TIMS 2011 International Study on Progress in Reading Comprehension, Mathematics and Sciences IEA. Volume II, Madrid, MECD, pp. 56-82.

GARCÍA, José M.; MARTínEZ-MONTEAgudo, María C. e INGLÉS, Candido J. (enero de 2013): "¿Cómo se relaciona la ansiedad con el rendimiento académico?", Revista lberoamericana de Psicología y Salud, no 4, vol.1, pp. 63-76.

HANUSHEK, Eric A.; MACHIN, Stephen y WOESSMANN, Ludger (2010): Handbook of the economics of education. Vol. 3. Amsterdam: Editorial North Holland.

INTERNATIONAL LABOUR ORGANIZATION (2012): International Standard Classification of Occupations: Structure, group definitions and correspondence tables, Geneve, International Labour Office, disponible en http://www.ilo.org/wcmsp5/groups/public/@dgreports/@dcomm/@publ/documents/publication /wcms 172572.pdf [consulta: 15 de junio de 2019].

INSTITUTO NACIONAL DE EVALUACIÓN EDUCATIVA (INEE) (2017): PIRLS 2016. Estudio Internacional de Comprensión Lectora. IEA. Informe Español. Madrid, MECD.

JOVELL, Albert J. (1995): Análisis de regresión logística, Madrid, Cuadernos Metodológicos, no 15, Centro de Investigaciones Sociológicas (CIS).

KHANDER, Shahindur R., LAVY, Victor y FILMER, Deon (1994) "Schooling and cognitive achievements of children in Morocco: Can the government improve outcomes?" World Bank Discussion Papers, The World Bank, Washington DC. https://doi.org/10.1596/0-8213-3046-2

LITTLE, Todd D.; JORGENSEN, Torrence D.; LANG, Kyle M. y MOORE, E. Whitney G. (marzo de 2014): "On the Joys of Missing Data", Journal of Pediatric Psychology, no 39, vol. 2, pp. 151-162. DOI: https://doi.org/10.1093/ipepsy/ist048

LITTLE, Roderick J. y RUBIN, Donald (2002): Statistical analysis with missing data, New York, Wiley. DOI: https://doi.org/10.1002/9781119013563.ch10 
LOZANO, Miguel A. y TRINIDAD, Antonio (febrero de 2019): “El Capital Cultural como Predictor del Rendimiento Escolar en España", International Journal of Sociology of Education, no 8, vol.1, pp. 45-74. http://dx.doi.org/10.17583/rise.2019.3862

MARTÍNEZ, José Saturnino (julio de 2017): “El habitus. Una revisión analítica” Revista Internacional de Sociología, no 75, vol. 3, pp. 1-14. http://dx.doi.org/10.3989/ris.2017.75.3.15.115

MARTIN, Michael O.; MULLIS, Ina V.S.; FOY, Pierre y HOOPER, Martin (2016): TIMSS 2015: International Results in Science, International Association for the Evaluation of Educational Achievement (IEA), disponible en http://www.educacionyfp.gob.es/inee/dam/icr:7bfa71d4-bef34efc-9bd3-a0db51dd3861/timss2015-international-results-in-science.pdf [consulta: 30 de mayo de 2019].

MEDINA, Fernando y GALVÁN, Marco (julio de 2007): "Imputación de datos: Teoría y práctica", Estudios estadísticos y prospectivos, no 54, División Estadísitca y proyecciones Económicas Naciones Unidas, CEPAL, disponible en https://repositorio.cepal.org/bitstream/handle/11362/4755/1/S0700590 es.pdf [consulta: 2 de mayo de 2020].

MORÉ, Iñigo: "El escalón económico entre vecinos. El caso España-Marruecos", Documentos de Trabajo, Real Instituto Elcano, 12 de diciembre de 2011, disponible en https://www.researchgate.net/profile/Im Martinez/publication/323143664_el_escalon_economi co entre vecinos/links/5a829fd8aca272d65019d318/el-escalon-economico-entre-vecinos.pdf [consulta: 12 de julio de 2019].

MULLIS, Ina V.S.; MARTIN, Michael O.; FOY, Pierre y HOOPER, Martin (2016): TIMSS 2015: International Results in Mathematics, International Association for the Evaluation of Educational Achievement (IEA), disponible en http://www.educacionyfp.gob.es/inee/dam/jcr:e650c54d-23154467-8edc-e32b0643527b/timss2015-international-results-in-mathematics.pdf [consulta: 30 de mayo de 2019].

MULLIS, Ina V.S.; MARTIN, Michael O.; FOY, Pierre y HOOPER, Martin (2017): PIRLS 2016: International Results in Reading, International Association for the Evaluation of Educational Achievement (IEA), disponible en https://www.educacionyfp.gob.es/inee/dam/jcr:16027373-dfd04005-a318-6f6d5d040a81/INFORME\%20INTERNACIONAL\%20PIRLS\%202016.pdf [consulta: 30 de mayo de 2019].

ORGANISATION FOR ECONOMIC CO-OPERATION AND DEVELOPMENT (OECD) (1999): Classifying Educational Programmes: Manual for ISCED-97 Implementation in OECD, Paris, OECD Publishing, disponible en http://www.oecd.org/education/skills-beyond-school/1962350.pdf [consulta: 20 de mayo de 2019].

OXFAM (2019): Un Maroc égalitaire, une taxation juste, disponible en https://www.oxfam.org/fr/rapports/un-maroc-egalitaire-une-taxation-juste [consulta: 20 de mayo de 2019].

PARDO, Antonio y RUIZ, Miguel A. (2013): Análisis de datos en ciencias sociales y de la salud III, Madrid, Síntesis.

PÉREZ, Carmen Nieves; BETANCORT, Moisés, y CABRERA, Leopoldo (enero de 2013): "Family influences in academic archievement a study of the Canary Islands", Revista Internacional de Sociología, no 71, pp. 169-187. https://doi.org/10.3989/ris.2011.04.11 
REY, P. (2012): Modelo para el tratamiento de conjuntos completos con datos ausentes de variables categóricas en un contexto de e-democrácia. Aplicación a encuestas de opinión. Universidad Politécnica de Madrid, Madrid, disponible en http://oa.upm.es/14754/ [consulta: 5 de mayo de 2020].

ROBERT, Christian P. y CASELLA, George (2004): Monte Carlo Statistical Methods, New York, Springer.

ROSENTHAL, Robert (1991): Meta-analytic procedures for social research, Newbury Park, Sage. https://doi.org/10.4135/9781412984997

ROSNOW, Ralph L. y ROSENTHAL, Robert (septiembre de 2003): “Effect Sizes for Experimenting Psychologists", Canadian Journal of Experimental Psychology, no 57, vol. 3, pp. 221-237. http://dx.doi.org/10.1037/h0087427

ROYSTON, PATRICK (agosto de 2004): “Multiple imputation of Missing Values", Stata Journal, no 4, vol., pp. 227-241. https://doi.org/10.1177/1536867X0400400301

RUBIN, Donald B. (1976), "Inference and Missing Data", Biometrika, no 63, vol. 3, pp. 581-592. https://doi.org/10.1093/biomet/63.3.581

RUBIN, Donald B. (1987): Multiple imputationfor Nonresponse in Surveys, New York, Jhon Wyley \& Sons. https://dx.doi.org/10.1002/9780470316696

SCHAFER, Joseph L. (febrero de 1999): "Multiple imputation: a primer", Statistical Methods in Medical Research, vol. 8, no 1, pp. 3-15. https://doi.org/10.1177/096228029900800102

SORIANO-MIRAS, Rosa M.; TRINIDAD, Antonio y KOPINAK, Kathryn (2015): "Los efectos de los Programas de Ajuste Estructural en la desigualdad social interna: El caso de Marruecos y México", Praxis sociológica, no 19, pp. 15-38.

TRINIDAD, Antonio; SORIANO-MIRAS, Rosa M., y SOLÍS, Marlene (2019): “Introduction: Global Localized Economies in a Comparative Perspective", en TRINIDAD, Antonio; SORIANO-MIRAS, Rosa M.; SOLÍS, Marlene y KOPINAK, Kathryn (Eds.): Localized Global Economies on the Northern Borderlands of Mexico and Morocco Cham, Palgrave Macmillan, pp. 1-12. https://doi.org/10.1007/978-3-319-96589-5 1

\section{Anexo I: Metodología de la Imputación múltiple empleada.}

Un análisis exploratorio de los datos nos aporta dos conclusiones importantes: las variables independientes están relacionadas entre sí y con la variable dependiente, como se verá en los siguientes análisis, y los datos perdidos de una variable no se concentran en zonas concretas de las distribuciones de otras variables, debido a que en el cálculo del chi-cuadrado de Little (Allison, 2001; Little y Rubin, 2002) obtenemos en todos los análisis de las nueve variables (no se ha tenido en consideración Ratio de alumnado por clase debido a que la población y la muestra son diferentes, como se ha indicado anteriormente) un $p$-valor superior de 0,1. Siguiendo la clasificación propuesta por Rubin (1976) los datos son perdidos al azar (MAR, por sus siglas en inglés) y siguiendo la caracterización de esta misma clasificación realizada por Little et al. (2014) son completamente al azar (MCAR). Por estas razones dos son las opciones más propicias para realizar la imputación: imputación múltiple (MI por sus siglas en inglés) y el método de máxima verosimilitud con información completa (FMIL) (Allison, 2001). La mayor diferencia entre ambas es que el FMIL no se debe utilizar a posteriori cuando los datos perdidos son MAR (Enders, 2010; Rey, 2012), ya que las variables predictoras han de ser diseñadas con tal propósito antes del trabajo de campo. 
Para predecir las seis variables con casos perdidos imputados hemos elegido las siguientes variables auxiliares: Actv_Temprns, Sexo e Inmigración. La técnica seleccionada para introducir los valores son regresiones, líneales para imputar los casos en variables numéricas y logísticas basadas en dummys para las politómicas. Sin embargo, como señala Allison (2001) los valores imputados poseerían muy poca varianza. Para solucionar este problema a las regresiones se le ha sumado el producto de la desviación típica estimada (s) y un valor seleccionado aleatoriamente $(E)$.

$$
\widehat{\mathrm{Z}}=b_{0}+b_{1} X+b_{2} Y+b_{3} H+s E
$$

Sin embargo, siguiendo con el planteamiento de Allison (2001) E introduciría diversas disfunciones derivadas de incorporar variabilidad muestral, por ello se hace necesario estimar varios valores por cada uno que pretendamos imputar y, por consiguiente, obtener varias bases de datos con los valores perdidos imputados. Para ello Rubin (1987) aportó una fórmula para estimar el error típico $(E E)$ de la media de parámetro de interés $(\bar{a})$ :

$$
E E(\bar{a})=\sqrt{\frac{1}{M} \sum_{k=1}^{M} S_{k}^{2}+\left(1+\frac{1}{M}\right)\left(\frac{1}{M-1}\right) \sum_{k=1}^{M}\left(a_{k}-\bar{a}\right)^{2}}
$$

Donde $a$ es el error estándar de la media del parámetro de interés. En nuestro caso el número de bases de datos generados $(M)$ es 25 . Royston (2004) defiende que 20 son suficientes. Además, para la variable que más casos perdidos acumula (Ocup_PyM en Marruecos $L P$ ) obtenemos un $98,9 \%$ de eficacia relativa de $M$ de Rubin $(p .114,1987)$. Además, $k$ es el error típico de cada base de datos y $S_{k}$ la estimación para cada parámetro $\left(a_{k}\right)$.

Por ello hemos utilizado el método estocástico de Monte Carlo y cadenas de Markov (MCMC) de especificación totalmente condicional para estimar las simulaciones siguiendo la metodología propuesta por Schafer (1999), la cual se encuentra esquematizada por Medina y Galván (p. 32-33, 2007), lo que asegura ciertas regularidades en la distribución límite de las diferentes bases de datos simuladas (Robert y Casella, 2004). El número de iteraciones ha sido 20, suficiente para que haya convergido.

Para realizar los análisis logit hemos optado por analizar conjuntamente las 25 bases de datos ya imputadas, tal y como recomiendan Medina y Galván (2007). 